\title{
A Clinical Shoulder Proprioception Test Shows Good Reliability, and Decreased Shoulder Joint Position Sense Due to Local Fatigue in Handballers: A Repeated Measures Design
}

\section{Peter Sutton}

Karlstad Medical Training Institute

Marie Ohlsson

Mid Sweden University

Ulrik Röijezon ( $\nabla$ ulrik.roijezon@ltu.se )

Luleå University of Technology

\section{Research Article}

Keywords: fatigue, gleno-humeral joint, handball, joint position sense, laser pen, proprioception, reliability, validity

Posted Date: April 28th, 2021

DOI: https://doi.org/10.21203/rs.3.rs-459791/v1

License: (c) (1) This work is licensed under a Creative Commons Attribution 4.0 International License.

Read Full License 


\section{Abstract}

Background: Assessment of shoulder proprioception outside the laboratory in the absence of specialized equipment remains a challenge with field-based tests often lacking good reliability, validity and feasibility. This study aimed to enhance the knowledge base surrounding the assessment of shoulder proprioception and investigated the effect of fatigue on shoulder joint position sense (JPS) amongst amateur male handball players.

Method: 27 healthy recreationally active participants and 13 amateur male handball players undertook two sessions of active JPS tests using laser pen technology and a calibrated 2-dimensional target to assess test-retest reliability. The active JPS test was then utilized on the subgroup of handball players who were subjected to five bouts of a repeated throwing task in order to investigate the effect of local fatigue on shoulder JPS.

Results: The intraclass correlation coefficient for the active JPS test was $0.78(95 \% \mathrm{Cl}=[0.57 ; 0.89])$. Standard error of measurement between trials was $0.70^{\circ}$ (range $0.57^{\circ}-0.90^{\circ}$ ). For the throwing task, repeated measures analysis of variance revealed a significant interaction for arm $x$ bout ( $F 5=2.74$, $p=0.028)$ and a significant effect for arm ( $F 1=5.85, p=0.034)$. Post hoc analysis showed a significant difference between throwing arm and non-throwing arm after throwing bout one $(p=0.036)$, three $(p=0.026)$ and four $(p=0.041)$.

Conclusion: Assessment of the active JPS test showed acceptable reliability and measurement error. Repeated throwing to fatigue decreased shoulder JPS in amateur male handball players which indicate validity of the test to identify reduced proprioception.

\section{Background}

Although there is a requirement for proprioceptive training and objective testing amongst athletes and in the clinical setting, the assessment of proprioception in the shoulder has traditionally been a challenge [1]. To date there have been wide variations in methodology and populations sampled with poor psychometric properties reported in tests [2]. Emerging field based tests often lack reliability and validity [3], however laboratory assessment methods with greater precision and psychometric properties are often not available for the everyday therapist wanting to assess proprioception.

Proprioception includes joint position sense (positional awareness of body parts and angles), kinesthesia (awareness of movement) and sensation of force $[2,4]$ and occurs through the stimulation of mechanosensory neurons (termed proprioceptors) located in the tissues providing afferent feedback [1, 4, 5]. Through the complex integration of peripheral and central systems [1, 6], the sum of these proprioceptors is important for both reactive and preparatory control as well as regulating muscle stiffness [4] in order to achieve movement coordination, timing and joint stability [7]. Being the most mobile and inherently unstable joint in the human body [8], the requirement for a strong and coordinated active stabilizing system in the shoulder joint is imperative. Consequently, any event negatively impacting 
neuro, muscular and skeletal systems has the potential to decrease proprioception [7], whether that be due to pain, effusion, trauma or fatigue [4]. Studies of the shoulder have shown a reduction in proprioception amongst subjects with joint laxity [9], in athletes undertaking overhead sports [10], in conjunction with shoulder pain [11], rotator cuff injuries [12] and amongst athletes who are fatigued [7, 13-16]. Although the role of proprioception in musculoskeletal disorders has been extensively researched in the lumbar and cervical spine [17-19] comparatively, there is limited research within the shoulder complex.

With the handball shoulder exposed to on average 48,000 throwing motions per season [20], 3-12 shots on goal and up to 180 ball contacts per match [21,22], the effect of fatigue on proprioception has a good theoretical base. The extent to which this can be explained through central or peripheral mechanisms however requires further investigation. Peripherally, concentric contraction can lead to fatigue through the depletion of metabolic factors with eccentric contractions resulting in both metabolic fatigue and muscle damage [23]. It has also been proposed that fatigue increases the threshold of muscle spindle discharge effecting afferent feedback [24]. Within the central nervous system, central fatigue can potentially inhibit proprioception through diminished motor control and muscle stabilizing activity [24]. The effect of central and peripheral fatigue on shoulder proprioception has been investigated $[22,25]$ and although this research offers further insight into proprioception, it paradoxically highlights the complexity of peripheral and central mechanisms underpinning a local response.

Contemporary research $[3,26]$ investigating joint position sense (JPS) has included the use of laser pens allowing the assessment of athletes with a field-based test. Balke et al [3] initially used laser technology to evaluate the proprioceptive deficit in patients with shoulder instability through the use of an active JPS test however did not evaluate the reliability and validity of the test. Glendon and Hood [5] subsequently amended the methodology and showed no difference between dominant and non-dominant arms in healthy subjects but also recommended further studies to establish inter and intra-rater reliability. This concept was subsequently developed further by Vafadar et al. [26] who investigated the inter-rater, intrarater reliability and validity of an active JPS test. They concluded inter-rater and intra-rater intraclass correlation coefficients were .86 and .78 respectively for this assessment method and the test had good validity when validated against a Vicon motion-capture system (Vicon, Oxford Metrics Ltd, Oxford, UK). The barrier for clinicians using this method, however, is that although the collection of data is efficient, the calculation of angle error afterwards remains time consuming. The aim of this study was to evaluate the test-retest reliability of an active JPS test using a laser pen and calibrated two-dimensional (2D) target for swift and feasible calculation of reposition error. Another aim was to investigate the effect of local fatigue, due to repeated throwing, on JPS in amateur male handball players.

\section{Method}

\section{Design}

In this study we used a test-retest and an experimental repeated measures design. 


\section{Participants}

Forty participants ( 15 women, 25 men) were recruited for the assessment of test-retest reliability. This included twenty-seven healthy recreationally active participants and thirteen amateur male handball players who subsequently also participated in the second part of the study. Participants were aged between 18 and 50 (mean 34.4 years $(S D \pm 8.4)$ ) and had a mean height of $177.7 \mathrm{~cm}( \pm 9.2 \mathrm{~cm})$. Hand dominance was assessed using the Edinburgh handedness inventory [27] and accordingly one participant was left handed, thirty-eight were right handed and one participant was ambidextrous. The thirteen healthy amateur male handball players were deemed eligible to participate in the throwing task. Players were aged between 18 and 34 (mean 26 (SD \pm 4.6 years)) with a mean height of $184.1 \mathrm{~cm}( \pm 4.2$ $\mathrm{cm})$, mean weight $91.7 \mathrm{~kg}( \pm 10 \mathrm{~kg})$ and had an average playing experience of 14.9 years ( \pm 4.6 years). One player was left handed and the remaining participants were right handed. Participants were included if they reported no history of shoulder injury or if they had returned to competition following an injury and were deemed to be subjectively medically fit. Exclusion criteria for both parts of the trial were current impairment of the shoulder (gleno-humeral or scapulathoracic joint), cervical, thoracic or lumbar spine, if they reported any neurological, systemic or rheumatological dysfunction or if they had any musculoskeletal impairment that would affect their ability to undertake the test. Data collection for 27 participants occurred at a medical training institute with the 13 handballers tested at their handball training facility. Testing of the handball players occurred 48 hours after the last match or training session

to allow adequate recovery [14] and was carried out by a Chartered Physiotherapist. Ethical approval was granted from the Swedish Ethical Review Authority (ref nr: 2019-06368) and written consent was obtained from the participants. All procedures and methods were performed in accordance with the guidelines and regulations of the Declarations of Helsinki.

\section{Equipment and set up for the active JPS}

A tennis elbow support (Mediroyal Nordic AB, Sweden) with a laser pointer (Zhongshan He Tong Optics Electronic Technology LTD, China) attached to the side was strapped to the lateral side of the humerus of participants just proximal to the lateral epicondyle on their dominant arm. Participants sat on a stool without backrest in front of a laminated moveable two dimensional (2D) A3 sized calibrated bullseye target. The participant lifted their dominant arm up to $90^{\circ}$ of flexion whilst keeping their elbow extended and wrist in a neutral position with thumb pointing towards the ceiling. This angle was measured with a goniometer to assure $90^{\circ}$ in accordance with Sawant et al [28]. Whilst maintaining this angle, the target was moved so that the laser dot was pointing at the centre of the target. The participant was then positioned so that the distance from the end of the laser pointer to the centre of the target was $90 \mathrm{~cm}$. For the participants undertaking the throwing task afterwards, this same procedure was then repeated for the non-dominant arm whereby a second target was utilized.

\section{Active JPS test}

The test was performed as an active-active JPS test with participants blindfolded. On command, the participant raised their dominant arm at a comfortable speed to $90^{\circ}$ and at this stage the examiner 
assisted their shoulder so that the laser was pointing at the centre of the target. When assisting the participant, the examiner held one hand on their forearm and when nearing the target centre, a small oscillation in the sagittal and then transverse plane was undertaken by the examiner in order to reduce a possible learning effect with the final position resulting in the laser pen pointing at the centre of the target. The participant was instructed to memorize this position for three seconds before lowering their arm to the starting position and then raising it at a self-chosen velocity back to the bullseye. When they felt they had raised their arm to the remembered position, they said 'yes' and the angle from the $2 \mathrm{~d}$ chart was documented (see Fig. 1). Participants subsequently repeated four more repetitions, with the examiner assisting the participant's shoulder to $90^{\circ}$ each time in conjunction with a small oscillation. Angle documentation occurred through documenting the laser dot on the target (angles on the target ranged from $\left.0-9^{\circ}\right)$. If the reproduction angle error exceeded the targets maximal error angle of $9^{\circ}$, the angle of error was calculated using trigonometry with $\tan 0$ (shoulder angle) = opposite side/adjacent side, where the opposite side was the distance from the recorded laser dot and centre of target and the adjacent side was the distance from the laser pointer to the laser dot. Absolute error (AE) was calculated as the mean of the five trials for each test occasion.

\section{Test-retest reliability}

For the assessment of test-retest reliability, participants undertook five repetitions of the active JPS test on their dominant arm. They then rested ten minutes before repeating the test. During this ten-minute period participants were allowed to leave the test position if they desired.

\section{Throwing task}

Thirteen handballers who had completed the test-retest reliability trial subsequently participated in the second part of the study thereafter. After undertaking their normal warm up protocol, participants were passed a standard men's handball (size 3 , circumference $58-60 \mathrm{~cm}$, weight $425-475 \mathrm{grams}$ ) from the test leader before advancing to the free throw line (9 metres from the goal) and threw the ball towards the goal with their dominant hand. Participants were instructed to throw the ball at maximum speed with a focus on force production as opposed to accuracy. Participants undertook ten throws with a maximum of ten seconds between each throw and were asked to complete five bouts of the throwing protocol, with a bilateral active JPS test and assessment of fatigue occurring directly after each throwing bout.

\section{BORG scale}

After each throwing bout of ten throws, participants were asked to rate the level of perceived exertion (RPE) they experienced in their shoulder using the BORG scale (6-20) [29]. Fatigue was defined as reaching 15 or greater and was chosen as it has been shown to correlate with the metabolic responses of fatigue [30] and is in accordance with the assessment method used in previous research [16, 31].

\section{Data analysis}

Test-retest reliability was analysed with an intraclass correlation coefficient (ICC). ICC estimates and their 95\% confidence intervals were calculated using IBM SPSS Statistics for Macintosh (Version 25.0. 
Armonk, NY: IBM Corp) based on a mean rating, absolute agreement, 2 way mixed effects model [32]. For the premise of this study, values were interpreted as follows: less than $0.5=$ poor reliability, between 0.5 and $0.75=$ moderate reliability, between 0.75 and $0.9=$ good reliability, greater than $0.9=$ excellent reliability [32]. A paired t-test was undertaken comparing the test-retest data in order to investigate a possible learning effect. Standard error of measurement (SEM) was calculated using the following formula:

\section{$\mathrm{SEM}=\mathrm{s}_{\text {diff. }} / \sqrt{2}$}

Furthermore, the minimal detectable change (MDC) was calculated for test and re-test data using the following formula:

\section{MDC = Standard Error of Measurement $\times 1.96 \times \sqrt{2}$}

The effect of fatigue on JPS AE was analyzed with a repeated measures ANOVA using IBM SPSS Statistics for Macintosh (Version 25.0. Armonk, NY: IBM Corp). Data for shoulder (dominant and nondominant), throwing bouts, and arm $\mathrm{x}$ bout was analyzed for all six occasions, i.e., baseline and after each of the five throwing bouts, using a mixed between (shoulders) and within (bouts) measures design. Post hoc analysis with Fisher's least significant difference (LSD) was undertaken if the effects were significant for the repeated measures ANOVA.

\section{Results}

\section{Flow of participants through the study}

A flow chart of the procedures and participants is presented in Fig. 2.

\section{Test-retest reliability}

The test-retest assessment of the active JPS test revealed an ICC of 0.78 (CI95\% 0.57-0.89). The paired ttest showed a significant difference in the JPS test AE scores between first test $\left(3.27^{\circ} \pm 0.98^{\circ}\right)$ and the retest $\left(2.97^{\circ} \pm 0.78^{\circ}\right)(t(39)=2.605, p=0.013)$. The SEM between the two tests was $0.70^{\circ}\left(C 195 \% 0.57^{\circ}-\right.$ $0.90^{\circ}$ ) with an MDC of $1.9^{\circ}$. A scatterplot of the AE from the test and re-test is shown in Fig. 3.

\section{Effects of fatigue after throwing task}

Although thirteen players were tested, one player failed to complete the throwing protocol due to shoulder pain and subsequently data analysis occurred for the remaining twelve participants. Assessment of the homogeneity of variance of differences showed the data met the assumption of sphericity using the Mauchly's test. The repeated measures ANOVA revealed a significant interaction for arm $\mathrm{x}$ bout (F5 $=2.74$, $p=0.028)$ and a significant effect for arm ( $F 1=5.85, p=0.034)$ but not for bout $(F 5=0.27, p=0.930)$.

Post hoc analysis using pairwise comparisons showed a significant difference between the fatigued 
dominant arm and the non-fatigued non-dominant arm after throwing bout one $(p=0.036)$, three $(p=$ $0.026)$ and four $(p=0.041)$. Figure 4 shows data for each arm per test occasion.

\section{Rate of perceived exertion}

Subjective assessment of fatigue, assessed with the Borg RPE scale, showed a gradual increase in the level of fatigue after every throwing bout. Figure 5 shows the fatigue perceived by participants undertaking the throwing protocol.

\section{Discussion}

The aim of the study was to evaluate the intra-tester reliability of an active JPS test using a laser pen and calibrated 2D target as well as investigate the effect of repeated throwing on shoulder JPS amongst amateur male handballers. The results showed that assessment of JPS using the documented active JPS test had good reliability and repeated throwing to fatigue was shown to reduce shoulder JPS compared to the control arm.

\section{Test-retest reliability of the active JPS test}

The results showed that assessment of JPS using the documented active JPS test had an ICC of 0.78 and is in line with the findings of Vafadar et al. [26]. Similarly, our research showed a small SEM of $0.7^{\circ}$ and a MDC of $1.9^{\circ}$. These results also reflect the findings of Vafadar et al, [26] who had an SEM range of $0.6^{\circ}$ to $1.2^{\circ}$ and an MDC of $1.8^{\circ}$. The similarity in results potentially highlights the importance of laser pen placement as methodological variations has occurred between the other studies. Compared to Glendon and Hood [5] and Balke et al. [3] who positioned the laser pen on the wrist and index finger respectively, our placement occurred proximally and subsequently interference from the joints distal to the shoulder girdle was eliminated. Although placement of the laser pen was consistent with that of Vafadar et al. [26] our methodology differed slightly with the aim to improve the test and clinical feasibility. Participants in our study were seated in order to reduce any sway that has been previously acknowledged [26]. The current study also utilized a 2D bullseye target. This allowed for AE to be documented and interpreted instantly as opposed to retrospectively using trigonometry. This quick and efficient way of measuring angle error has benefits to clinicians utilizing this assessment method clinically as athletes and patients can get instant feedback on the results. We also incorporated an oscillatory relocation when relocating the shoulder to the target angle to minimize any training effect as has been reported to influence repeated tests [33].

Previously, a variety of methods and equipment have been used to assess shoulder JPS with the greatest intra-rater reliability being demonstrated when using isokinetic dynamometry (ICC 0.92) [1]. Utilizing the documented active JPS test is however similar to that of an inclinometer (ICC 0.84) photo analysis through retrospective angle calculation (ICC 0.81) and is superior to using a goniometer (ICC 0.60) [1]. Although assessment of shoulder and scapula proprioception in the laboratory [34] has shown a lower SEM the results in the current study are superior to those reported from Suprak et al. [35] (SEM range 1.9 ${ }^{\circ}$ 
to $4.07^{\circ}$ ) and Kaya et al. [36] (SEM range $3.87^{\circ}$ to $4.5^{\circ}$ ) who assessed shoulder JPS with digital tracking and isokinetic dynamometry respectively [1].

Although acknowledged that the interpretation of the ICC score varies considerably in the literature, the results were interpreted in accordance with the parameters recommended by Koo and Li [32]. The implications of this is that clinicians are able to assess shoulder JPS with good reliability using the documented methodology. The generalization of results however must be made with caution and further testing for inter-rater reliability is warranted prior to this assessment method being utilized across multiple clinicians for the same subject. Similarly, the effect of repeated testing at this stage is not fully unraveled. When participants were subjected to repeated testing after the throwing task, the reduction in $A E$ in the non-dominant non-fatigued shoulder started to plateau after throwing bout three (see Fig. 4). The implications of this are that athletes may need to undertake the active JPS test up to three times before any true JPS deficit can be identified. The most likely explanation for this change was the learning effect indicating a systematic bias [33]. This was also evident in the test-retest trial whereby greater JPS acuity was demonstrated in the retest $\left(-0.3^{\circ}, S D \pm 0.7\right)$. This occurred despite the methodology incorporating an oscillation when relocating the shoulder to minimize this effect, and should be considered and evaluated in future studies.

\section{Throwing task}

A reduction in JPS on the dominant arm from baseline to throwing bout five was demonstrated with statistical significance being reached after throwing bouts one, three and four compared to the nonfatigued non-dominant control shoulder. These findings reflect previous research investigating the role of fatigue on shoulder JPS $[7,13-15,31]$. Although it has been proposed that contemporary research can not determine which part of the proprioceptive pathway is responsible for the reduction in proprioceptive sense [37] the results in the throwing task add weight to the role of peripheral mechanisms in JPS diminshment. This is due to the fact that the dominant throwing shoulder showed an increase in AE over the testing bouts compared to the non-dominant control arm. With the throwing action encorporating both concentric and eccentric components in the rotator cuff, the depletion of metabolic factors and inflammatory substances through fatigue can possibly explain the disturbance to JPS witnessed [23]. In essence, these physiological changes can slow down muscle fibre conduction velocity and increase the level of neural activation required to produce force [38]. The effect of a large cumulative eccentric load in the dorsal rotator cuff muscles due to repeated throwing is important as they have been shown to demonstrate a lower fatigue resistance [39] and therefore any desensitisation of these tissues and posterior capsular receptors could subsequently lead to an increase in angle error [13]. In conjunction with this, the velocity of the throwing action would have biased the quick adapting mechanoreceptors (muscle spindle receptors) over the slower adapting joint receptors [13] directly impacting the discharge patterns of the muscle spindles and decreasing JPS further [24]. The high frequency throwing protocol may potentially also have created some muscle soreness in the shoulder altering proprioception through the group III and IV muscle afferents [23]. 
In comparison to handball matches [21], participants were exposed to a significantly higher throwing frequency within a short time frame reinforcing the impact of local fatigue in the shoulder complex. Subsequently the group reached a mean RPE of approximately 15 after throwing bout 4 . The impact of throwing influencing JPS via central fatigue can however not be ruled out. Possible mechanisms include diminished motor control and muscle stabilizing activity [24], failure to adequately drive motorneurons [40] and the central governor model of exercise regulation [41]. Investigating central fatigue however was beyond the scope of the trial. A further limitation to the study was the extent of fatigue in the underlying tissues. Electromyographic (EMG) studies of the shoulder [42] have shown the highest maximum voluntary isometric contraction to occur in the rotator cuff during overhead throwing. In the absence of muscle testing using dynamometry or EMG (changes in amplitude and frequency due to fatigue) the true extent of fatigue in the shoulder beyond individual perception cannot be quantified. Although laboratory studies $[16,31,43]$ can be deemed to have greater validity, the results can be difficult to extrapolate and generalize to clinical or sporting situations [24]. The ecological validity of our results, replicating the sporting demands, can potentially offer training advice as the link between fatigue, subtle instability and overuse injuries that has been proposed [38]. It could be considered more clinically relevant to assess shoulder JPS in handball players with an active-active relocation test occurring in abduction and rotation i.e replicating the throwing motion, which should be investigated in future studies. However, contemporary research $[3,5,26]$ using a laser pen has only been assessed in flexion. In that respect, we wanted to build on current knowledge and further develop a feasible, reliable and validated test when assessing shoulder JPS in general. It must be acknowledged however that JPS is only one of the elements of proprioception and further work is warranted to investigate the concept of proprioceptive impairment in the throwing shoulder for kinesthesia and sense of force.

\section{Conclusion}

Test-retest reliability of the shoulder active JPS test has been shown to have good reliability and is in keeping with previous research [26]. This test can therefore be used in the assessment of JPS clinically with acceptable reliability and is quick and feasible to perform. The findings of the throwing protocol demonstrate a reduction in JPS when the shoulder is fatigued. Coaches can potentially utilize the data on throws to fatigue to amend warm up protocols whereby players naturally have a high throwing frequency and upper limb bias before matches.

\section{Abbreviations}

\section{AE: Absolute error}

ICC: Intraclass correlation coefficient

JPS: Joint position sense

LSD: Least significant difference 
MDC: Minimal detectable change

RPE: Rate of perceived exertion

SEM: Standard error of measurement

2D: Two dimensional

\section{Declarations}

\section{Ethics approval and consent to participate:}

Ethical approval was granted from the Swedish ethical review committee (ref nr: 2019-06368). All participants gave written informed consent to participate in the study.

\section{Consent for publication:}

Written informed consent for publication of the clinical image in figure 1 was obtained. A copy of the consent form is available for review from the first named author of this journal.

\section{Availability of data and materials:}

The datasets used and analysed are available from the first named author on reasonable request but restrictions apply to the availability of these data, which were used under license for the current study.

\section{Competing Interests:}

The authors declare that they have no competing interests.

\section{Funding:}

This research did not receive any specific grant from funding agencies in the public, commercial or not-for-profit sectors.

\section{Authors' contributions:}

PS was responsible for data collection and writing the bulk of the manuscript. UR was the main supervisor and editor in this study. UR recommended, facilitated and was pivotal in all the statistical tests 
undertaken in this study. MO was co-supervisor and the first named authors go to supervisor whilst he was attending Mid Sweden University. All authors read and approved the final manuscript.

\section{Acknowledgements:}

None.

\section{References}

1. Ager AL, Roy JS, Roos M, Belley AF, Cools A, Hébert LJ. Shoulder proprioception: How is it measured and is it reliable? A systematic review. J Hand Ther 2017;30:221-231

2. Hillier S, Immink M, Thewlis D. Assessing Proprioception: A Systematic Review of Possibilities. Neurorehabil Neural Repair 2015;29:933-949

3. Balke M, Liem D, Dedy N, Thorwesten L, Balke M, Poetzl W, Marquardt B. The laser-pointer assisted angle reproduction test for evaluation of proprioceptive shoulder function in patients with instability. Arch Orthop Trauma Surg 2011;131:1077-1084

4. Röijezon U, Clark NC, Treleaven J. Proprioception in musculoskeletal rehabilitation: Part 1: Basic science and principles of assessment and clinical interventions. Man Ther 2015;20:368-377

5. Glendon K, Hood V. Upper limb joint position sense during shoulder flexion in healthy individuals: a pilot study to develop a new assessment method. Shoulder Elb 2016;8:54-60

6. Tuthill JC, Azim E. Proprioception. Curr Biol 2018;28:R194-R203

7. Herrington L, Horsley I, Whitaker L, Rolf C. Does a tackling task effect shoulder joint position sense in rugby players? Phys Ther Sport 2008;9:67-71

8. Lewis JS: Subacromial impingement syndrome: a musculoskeletal condition or a clinical illusion? Phys Ther Rev 2011;16:388-398

9. Laudner KG, Meister K, Kajiyama S, Noel B. The relationship between anterior glenohumeral laxity and proprioception in collegiate baseball players. Clin J Sport Med 2012;22:478-482

10. Safran MR, Borsa PA, Lephart SM, Fu FH, Warner JJP. Shoulder proprioception in baseball pitchers. J Shoulder Elb Surg 2001;10:438-444

11. Maenhout AG, Palmans T, De Muynck M, De Wilde LF, Cools AM. The impact of rotator cuff tendinopathy on proprioception, measuring force sensation. J Shoulder Elb Surg 2012;21:10801086

12. Gumina S, Camerota F, Celletti C, Venditto T, Candela V. The effects of rotator cuff tear on shoulder proprioception. Int Orthop 2019;43:229-235

13. Lee HM, Liau JJ, Cheng CK, Tan CM, Shih JT. Evaluation of shoulder proprioception following muscle fatigue. Clin Biomech 2003;18:843-847

14. Morgan R, Herrington L. The effect of tackling on shoulder joint positioning sense in semiprofessional rugby players. Phys Ther Sport 2014;15:176-180 
15. Myers JB, Guskiewicz KM, Schneider RA, Prentice WE. Proprioception and Neuromuscular Control of the Shoulder after Muscle Fatigue. J Athl Train 1999;34:362-367

16. Tripp BL, Boswell L, Gansneder BM, Shultz SJ. Functional Fatigue Decreases 3-Dimensional the Overhead-Throwing Athlete. J Athl Train 2004;39:316-320

17. Lin J, Halaki M, Rajan P LA. Relationship Between Proprioception and Pain and Disability in People With Non-Specific Low Back Pain: A Systematic Review With Meta-Analysis. Spine (Phila Pa 1976) 2019;44:E606-E617

18. Tong MH, Mousavi SJ, Kiers H, Ferreira P, Refshauge K, van Dieën J. Is There a Relationship Between Lumbar Proprioception and Low Back Pain? A Systematic Review With Meta-Analysis. Arch Phys Med Rehabil 2017;98:120-136.e2

19. Stanton TR, Leake HB, Chalmers KJ, Moseley GL. Evidence of impaired proprioception in chronic, idiopathic neck pain: Systematic review and meta-analysis. Phys Ther 2016;96:876-887

20. Pieper HG. Humeral torsion in the throwing arm of handball players. Am J Sports Med 1998;26:247253

21. Karcher C, Buchheit M. On-Court demands of elite handball, with special reference to playing positions. Sport Med 2014;44:797-814

22. Niederseer D, Mörtl H, Liebensteiner MC, Egger A, Thaler CW, Plöderl M, Niebauer J RC. General Fatigue and Joint Position Sense in Male Elite Handball Players. Ger J Sport Med 2014;65:149-153

23. Proske, Uwe, \& Gandevia SC. The proprioceptive senses: their roles in signaling body shape, body position and movement, and muscle force. Physiol Rev 2012;92:1651-1697

24. Fernando Ribeiro and José Oliveira. Biomechanics in Applications. Biomech Appl. https://doi.org/10.5772/20335

25. Freeston J, Adams R, Ferdinands RE RK. Indicators of throwing arm fatigue in elite adolescent male basketball players: a randomized crossover trial. J Strength Cond Res 2014;28:2115-2120

26. Vafadar AK, Côté JN, Archambault PS. Interrater and Intrarater Reliability and Validity of 3 Measurement Methods for Shoulder-Position Sense. J Sport Rehabil Technical: 2016;2014-0309

27. Veale JF. Edinburgh Handedness Inventory - Short Form: A revised version based on confirmatory factor analysis. Laterality 2014;19:164-177

28. Sawant A, Norkin CC WJ. Measurement of Joint Motion, A Guide to Goniometry. Physiother Canada 2004;56:250

29. Borg GA. Borg`s RPE Scale.pdf. Med Sci Sport Exerc Vol 1982;14:377-381

30. Kang J, Chaloupka EC, Mastrangelo MA, Donnelly MS, Martz WP, Robertson RJ. Regulating exercise intensity using ratings of perceived exertion during arm and leg ergometry. Eur J Appl Physiol Occup Physiol 1998;78:241-246

31. Rich RL, Struminger AH, Tucker WS, Munkasy BA, Joyner AB, Buckley TA. Scapular upward-rotation deficits after acute fatigue in tennis players. J Athl Train 2016;51:474-479 
32. Koo TK, Li MY. A Guideline of Selecting and Reporting Intraclass Correlation Coefficients for Reliability Research. J Chiropr Med 2016;15:155-163

33. Hopkins WG. Sports Medicine 30: 1-15, Jul 2000. Sport Med 2000;30:1-15

34. Deng HR, Shih YF. Test validity and intra-rater reliability in the measurement of scapular position sense in asymptomatic young adults. Man Ther 2015;20:503-507

35. Suprak DN, Osternig LR, Van Donkelaar P KA. Shoulder Joint Position Sense Improves with elevation angle in a novel, unconstrained task. J Orthop Res 2006;24:559-568

36. Kaya D, Callaghan MJ, Donmez G, Doral MN. Shoulder joint position sense is negatively correlated with free-throw percentage in professional basketball players. Isokinet Exerc Sci 2012;20:189-196

37. Abd-Elfattah HM, Abdelazeim FH, Elshennawy S. Physical and cognitive consequences of fatigue: A review. J Adv Res 2015;6:351-358

38. Coskun G, Talu B, Cools A. Proprioceptive force-reproduction of the rotator cuff in healthy subjects before and after muscle fatigue. Isokinet Exerc Sci 2018;26:175-181

39. Ellenbecker TS, Roetert EP. Testing Isokinetic Muscular Fatigue of Shoulder Internal and External Rotation in Elite Junior Tennis Players. 1999;29:275-281

40. Gandevia SC. Spinal and supraspinal factors in human muscle fatigue. Physiol Rev 20001;81:17251789

41. Noakes TD. Fatigue is a brain-derived emotion that regulates the exercise behavior to ensure the protection of whole body homeostasis. Front Physiol 2012;3 APR:1-13

42. RF E, JR A. Shoulder muscle recruitment patterns and related biomechanics during upper extremity sports. Sport Med 2009;39:569-590

43. Alizadehkhaiyat $\mathrm{O}$, Hawkes DH, Kemp GJ, Frostick SP. Electromyographic Analysis of the Shoulder Girdle Musculature During External Rotation Exercises. Orthop J Sport Med 2015;3:1-9

\section{Figures}




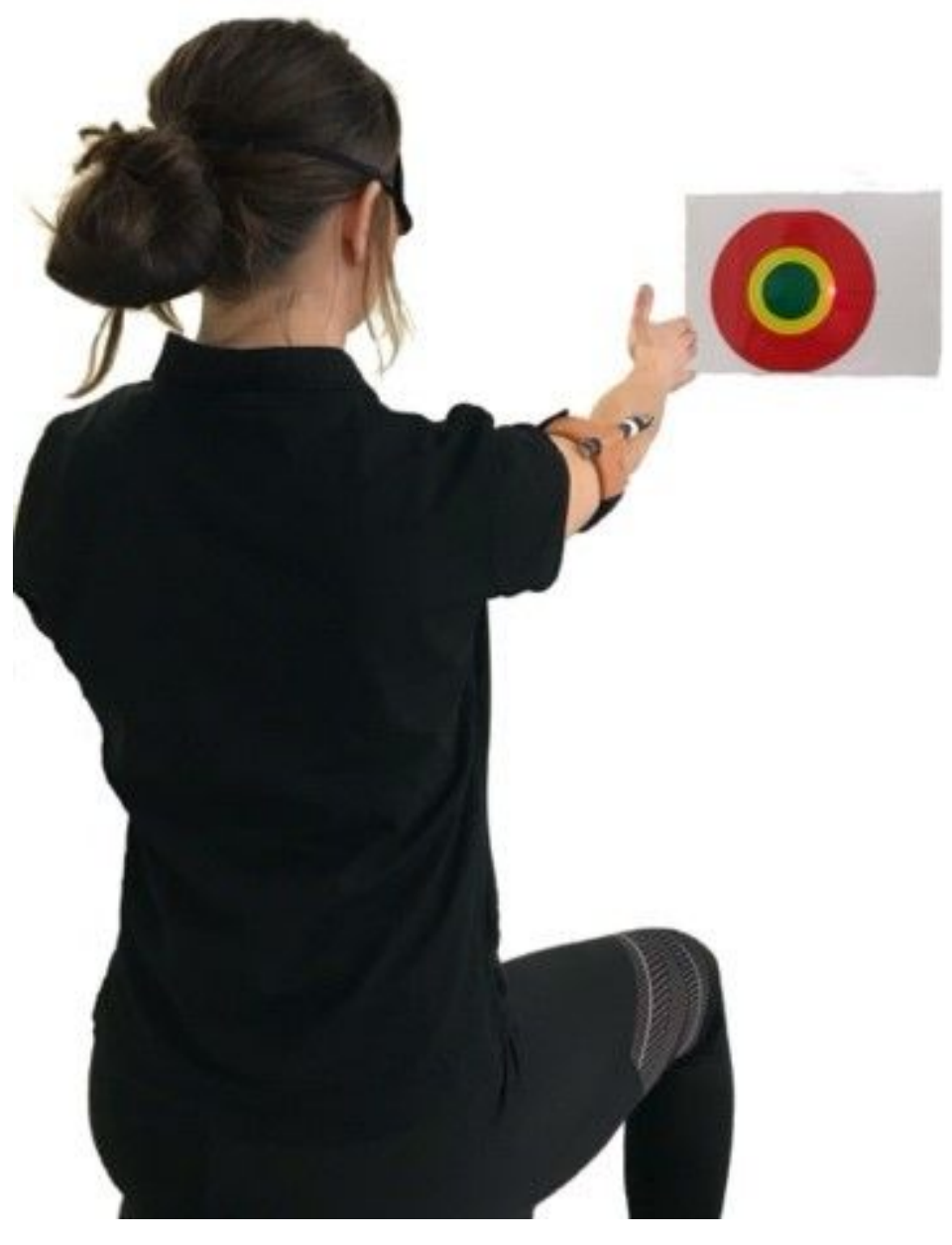

Figure 1

Joint position sense test of the shoulder. Illustration of the position of the participant, the laser pointer and target at the reposition angle during the test. 


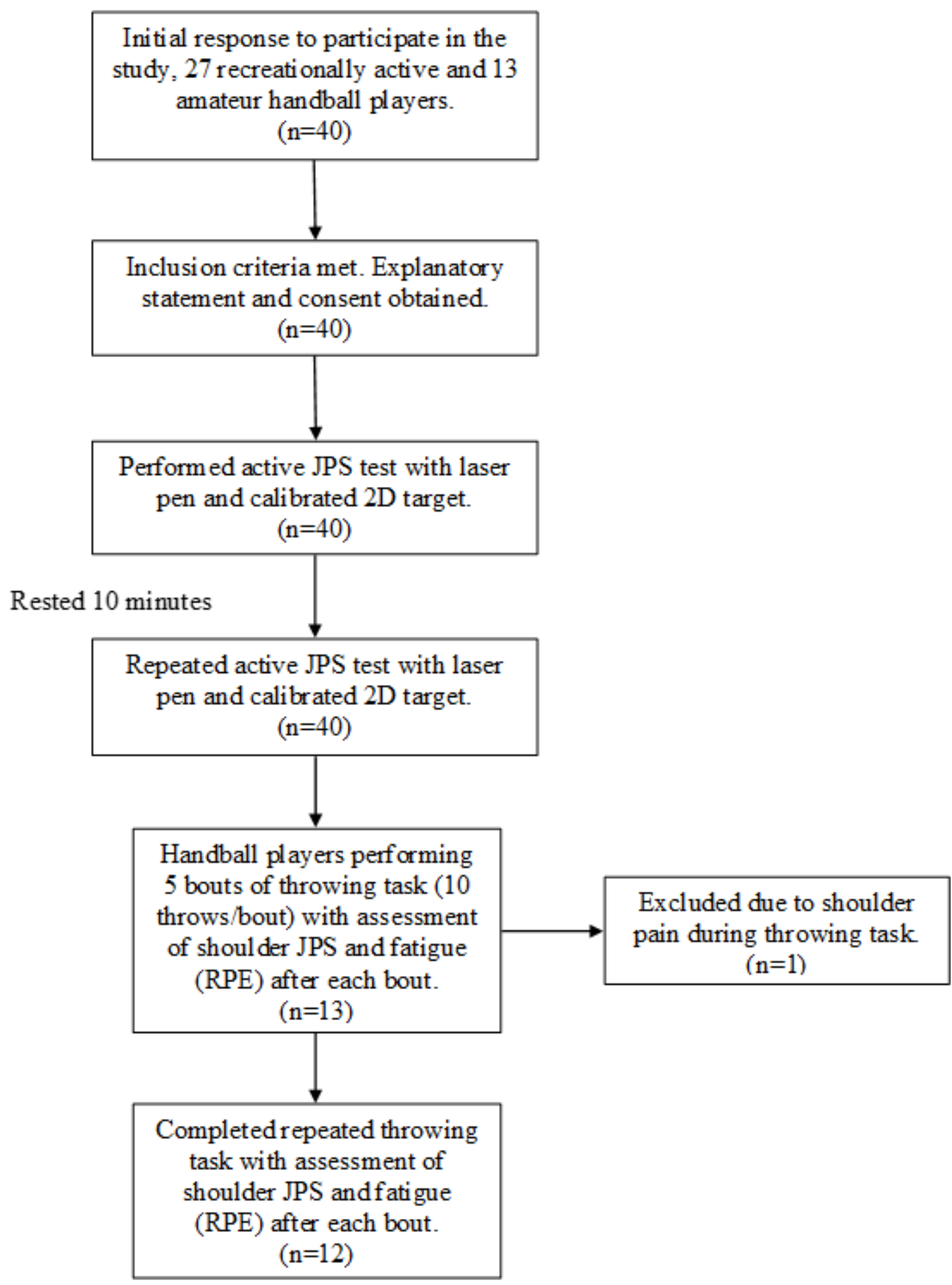

\section{Figure 2}

Flowchart of the study. Flow of participants through the study. JPS: joint position sense; 2D: two dimensional; RPE: rate of perceived exertion. 




Figure 3

Relationship between test 1 and 2. Scatterplot showing absolute error (AE) of the test-retest data with regression line 


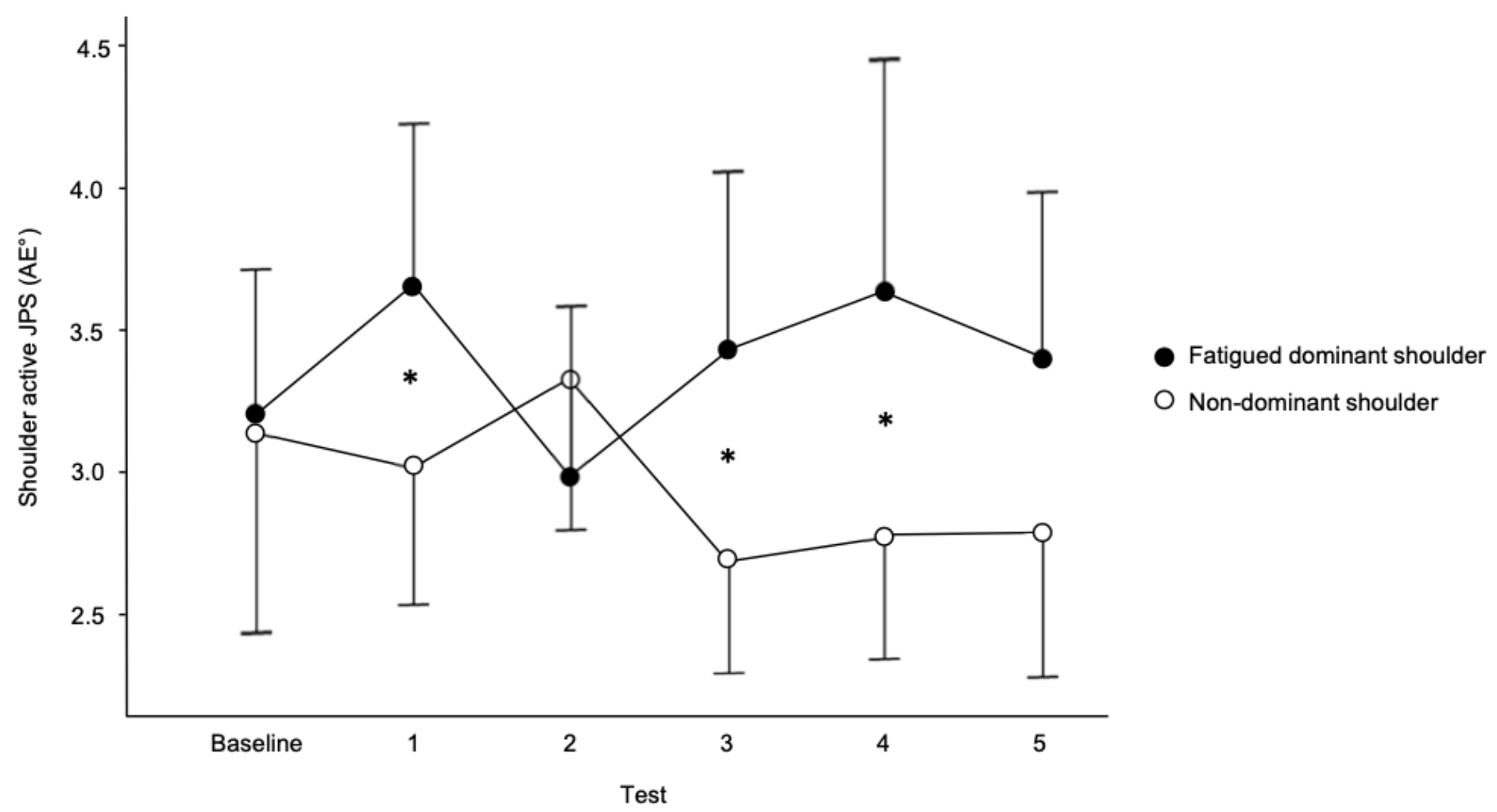

Figure 4

Proprioception difference between fatigued and non-fatigued shoulder. Line diagram showing mean values and $95 \%$ confidence intervals of the joint position sense (JPS) test absolute error (AE) for shoulders and repeated tests. ${ }^{*}=$ significant difference between dominant and non-dominant sides $p<0.05$ 


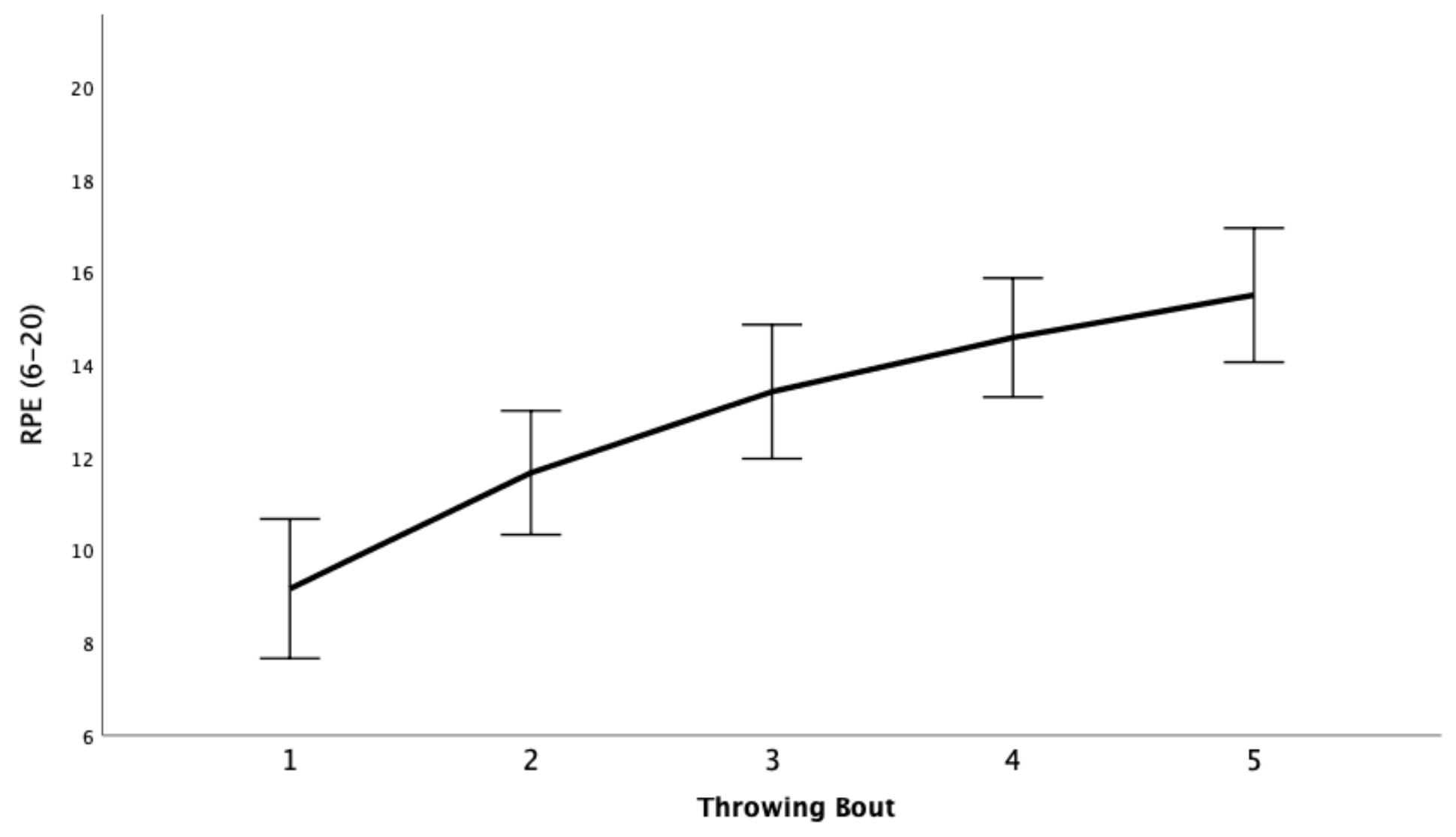

Figure 5

Perceived exertion after hand ball throwing task. Line diagram with trend line showing mean rate of perceived exertion (RPE) values and error bars showing $95 \%$ confidence intervals assessed immediately after each bout of ten consecutive high velocity handball throws. 